\title{
A NOTE ON THE NP-HARDNESS OF THE SEPARATION PROBLEM ON SOME VALID INEQUALITIES FOR THE ELEMENTARY SHORTEST PATH PROBLEM
}

\author{
M.S. Ibrahim ${ }^{1 *}$, N. Maculan ${ }^{2}$ and M. Minoux ${ }^{3}$ \\ Received September 27, 2012 / Accepted March 23, 2014
}

\begin{abstract}
In this paper, we investigate the separation problem on some valid inequalities for the $s-t$ elementary shortest path problem in digraphs containing negative directed cycles. As we will see, these inequalities depend to a given parameter $k \in \mathbb{N}$. To show the NP-hardness of the separation problem of these valid inequalities, considering the parameter $k \in \mathbb{N}$, we establish a polynomial reduction from the problem of the existence of $k+2$ vertex-disjoint paths between $k+2$ pairs of vertices $\left(s_{1}, t_{1}\right),\left(s_{2}, t_{2}\right) \ldots\left(s_{k+2}, t_{k+2}\right)$ in a digraph to the decision problem associated to the separation of these valid inequalities. Through some illustrative instances, we exhibit the evoked polynomial reduction in the cases $k=0$ and $k=1$.
\end{abstract}

Keywords: polytope, digraphs, shortest path, valid inequality, separation.

\section{INTRODUCTION}

Let $G=(V, E)$, be a connected directed graph and $s \in V$ and $t \in V$ two vertices of $G$. We suppose that $G$ contains $q$ elementary paths from $s$ to $t$, and we denote $p_{1}, p_{2}, \ldots, p_{q}$, these $s-t$ elementary paths. $V\left(p_{i}\right)$ and $E\left(p_{i}\right)$ are the set of vertices and the set of arcs corresponding to the $s$ - $t$ elementary path $p_{i}$ respectively.

Given a parameter $k \in \mathbb{N}$, let $\left(S_{k}, A_{k}\right)$ be a pair of sets $S_{k} \subset V$ and $A_{k} \subset E$ in the digraph $G=(V, E)$ such that:

- No arc in $A_{k}$ has an endpoint in $S_{k}$;

- $\forall p_{i}(i=1, \ldots, q),\left|\left(S_{k} \cap V\left(p_{i}\right)\right)\right|+\left|\left(A_{k} \cap E\left(p_{i}\right)\right)\right| \leq k$.

In Ibrahim (2008) and Ibrahim et al. (2014), we call a pair $\left(S_{k}, A_{k}\right)$ as defined above a $k$-subset pair with respect to vertices $s$ and $t$. An element of $S_{k}$ is called a $k$-vertex and an element of $A_{k}$ is called a $k$-arc.

\footnotetext{
*Corresponding author

${ }^{1}$ Université A. Moumouni, Faculté des Sciences, BP 10.622, Niamey, Niger. E-mail: ibrah_dz@yahoo.fr

${ }^{2}$ Federal University of Rio de Janeiro, Rio de Janeiro, RJ, Brazil. E-mail: maculan@ cos.ufrj.br

${ }^{3}$ Université Pierre et Marie Curie, Paris, France. E-mail: michel.minoux@lip6.fr
} 
For any pair $\left(S_{k}, A_{k}\right)$ which is a $k$-subset pair, the inequality

$$
\sum_{p \in S_{k}} x_{p}+\sum_{(q, r) \in A_{k}} y_{q r} \leq k
$$

is shown to be valid for $\mathcal{P}$, the polytope induced by the $s-t$ elementary paths in $G . x_{p}$ and $y_{q r}$ are binary variables associated to the vertex $p$ and the arc $(q, r)$ respectively, (see Ibrahim (2008), Ibrahim et al. (2014)). Such valid inequality is called a valid inequality of order $k$.

In this paper, we investigate the separation problem of the so-called valid inequalities of order $k$, first presented and exploited in cutting plane framework in view to solve the shortest path problem in digraphs possibly having negative cycles (see Ibrahim (2008), Ibrahim et al. (2014)). Considering a mixed integer linear model of the shortest elementary path problem, we use these valid inequalities in a cutting plane algorithm to build strong linear relaxations. For the mixed integer linear model of the shortest elementary path problem, one could refer to Maculan et al. (2003) and Ibrahim et al. (2009). We prove the NP-hardness of the separation problem of valid inequalities of order $k$ by establishing a polynomial reduction from the problem of the existence of $k+2$ vertex-disjoint paths between $k+2$ pairs of vertices $\left(s_{1}, t_{1}\right),\left(s_{2}, t_{2}\right), \ldots,\left(s_{k+2}, t_{k+2}\right)$ in a digraph to the decision problem associated to the separation of these valid inequalities.

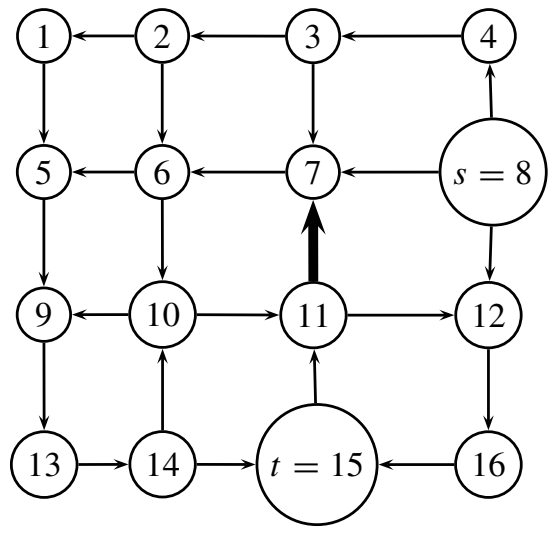

Figure 1 - $(11,7)$ is a 0 -arc w.r.t. the vertices

$$
s=8 \text { and } t=15 \text {. }
$$

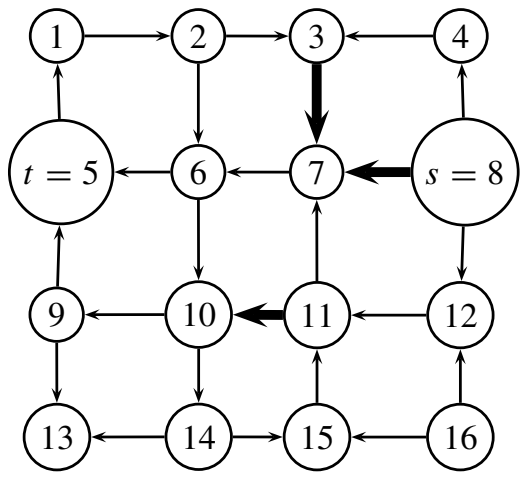

Figure $2-(11,10)$ is a 1 -arc w.r.t. the vertices $s=8$ and $t=5$.

Figure 1 and Figure 2 illustrate the concept of $k-\operatorname{arc}$ and $k-$ vertex. In Figure 1 , the arc $(11,7)$ is a $0-\operatorname{arc}$ w.r.t. vertices $s=8$ and $t=15$ as it is not belonging to any elementary path between vertices 8 and 15. In Figure 2, $\left(\emptyset, A_{1}\right)$ constitutes a 1-subset pair, with $A_{1}=$ $\{(3,7),(8,7),(11,10)\}$ and induces the valid inequality $y_{3,7}+y_{8,7}+y_{11,10} \leq 1$. That is, all $s-t$ elementary paths in Figure 2 passe by at most one of the $1-\operatorname{arcs}(3,7),(8,7)$ and $(11,10)$. One can also observe in Figure 2 that the vertices 14 and 15 are $0-$ vertices. $k-\operatorname{arcs}$ and $k$-vertices induce what we call valid inequalities of order $k$ and we will see that in general the problem consisting to detect such vertices and arcs is a difficult task. 
The paper is organized as follows. In section 2, we address some cases for which the separation problem of valid inequalities of order $k$ can be solved in polynomial time. Then, we show the NPhardness of the problem of separation of these valid inequalities in digraphs for a given general $k$. In section 3, considering the cases $k=0$ and $k=1$, we present some instances illustrating the evoked polynomial reduction between the problem of the existence of $k+2$ vertex-disjoint paths between $k+2$ pairs of vertices $\left(s_{1}, t_{1}\right),\left(s_{2}, t_{2}\right), \ldots,\left(s_{k+2}, t_{k+2}\right)$ and the decision problem associated to the separation of these valid inequalities in a digraph.

\section{SEPARATION PROBLEM FOR VALID INEQUALITIES OF ORDER $k$}

Given $(\bar{x}, \bar{y})$ an optimal (fractional) solution of a shortest path linear model, the separation problem w.r.t. valid inequalities of order $k$ consists in finding in $G$, a $k$-subset pair $\left(S_{k}, A_{k}\right)$ such that:

$$
\sum_{p \in S_{k}} \bar{x}_{p}+\sum_{(q, r) \in A_{k}} \bar{y}_{q r}>k .
$$

The problem of separating valid inequalities of order $k$ corresponds in looking for a $k$-subset pair $\left(S_{k}, A_{k}\right)$ in $G$ such that the valid inequality of order $k$ is violated.

\subsection{Some polynomial cases}

We have polynomial algorithms for some special cases:

- if $\Gamma^{+}(\alpha)=\emptyset$ or $\Gamma^{-}(\alpha)=\emptyset$, then $\alpha \in S_{k}, k=0$;

- if $t \in \Gamma^{+}(\alpha)$ and there is no elementary path from $s$ to $\alpha$, then $\alpha \in S_{k}, k=0$;

- if $s \in \Gamma^{-}(\alpha)$ and there is no elementary path from $\alpha$ to $t$, then $\alpha_{i} \in S_{k}, k=0$.

Where $\Gamma^{-}(\alpha)$ and $\Gamma^{+}(\alpha)$ denote the sets of arcs coming into and going out of the vertex $\alpha$, respectively. That is, considering a digraph $G=(V, E), \Gamma^{-}(\alpha)=\{(\beta, \alpha):(\beta, \alpha) \in E\}$ and $\Gamma^{+}(\alpha)=\{(\alpha, \beta):(\alpha, \beta) \in E\}$.

In the case of undirected graphs, for $k=0$, the first polynomial algorithms solving the problem of the existence of $k+2$ vertex-disjoint elementary paths between $k+2$ pairs of vertices $\left(s_{1}, t_{1}\right),\left(s_{2}, t_{2}\right), \ldots,\left(s_{k+2}, t_{k+2}\right)$ are due to Ohtsuzi (1981), Seymour (1980), Shiloach (1980) and Thomassen (1985). Robertson \& Seymour (1995) treat the general case of $k$.

In digraphs, there exist particular cases for which the problem of the existence of $k+2$ vertexdisjoint elementary paths between $k+2$ pairs of vertices $\left(s_{1}, t_{1}\right),\left(s_{2}, t_{2}\right), \ldots,\left(s_{k+2}, t_{k+2}\right)$ is solvable in polynomial time. Perl \& Shiloach (1978) present a polynomial algorithm that solves such problem, with $k=0$, in three connected directed planar and directed acyclic graphs. The latter result concerning directed acyclic graphs is extended for a given $k$ by Fortune et al. (1980). One can remark that in directed acyclic graphs, the separation problem of these inequalities is not interesting, as in such digraph the shortest path problem can be solved easily. On other hand, Schrijver (1994) present a polynomial method for planar digraphs for a given $k$. 


\subsection{The general case for the problem}

In the general case, for a given $k \in \mathbb{N}$, the separation problem consists in finding $k+1$-uplet $\left(\theta_{1}, \theta_{2}, \ldots, \theta_{k+1}\right)$ in $\mathrm{G}$ such that $\theta_{i} \in S_{k}$ or $\theta_{i} \in A_{k}$ and $i=1, \ldots, k+1$. If $\theta_{i} \in S_{k}$, we set $\theta_{i}=\alpha_{i}$, otherwise $\theta_{i} \in A_{k}$ and we set $\theta_{i}=\left(\alpha_{i}, \beta_{i}\right)$, where $\alpha_{i}$ and $\beta_{i}$ are the endpoints of the $\operatorname{arc} \theta_{i}$.

Let $\Pi_{k}$ be the following decision problem associated to the separation problem of valid inequalities of order $k$ :

"Given $k+1$ vertices and/or $\operatorname{arcs} \theta_{1}, \theta_{2}, \ldots, \theta_{k+1}$ in $G$, is $\theta_{i}$ a $k$-vertex or a $k$-arc?" With $i=1, \ldots, k+1$.

Consider the problem $\Pi_{k}^{\prime}$ defined as follow:

"Given $2 k+4$ distinct vertices $s_{1}, t_{1}, s_{2}, t_{2}, \ldots, s_{k+2}, t_{k+2}$, are there no $k+2$ elementary paths, $P_{s_{1}, t_{1}}, P_{s_{2}, t_{2}} \ldots P_{s_{k+1}, t_{k+1}}, P_{s_{k+2}, t_{k+2}}$ in G such that $V\left(P_{s_{i}, t_{i}}\right) \cap V\left(P_{s_{j}, t_{j}}\right)=\emptyset, 1 \leq i<j \leq k+2$ ?" Where $V\left(P_{s_{i}, t_{i}}\right)$ are vertex sets of the elementary path $P_{s_{i}, t_{i}}$ between $s_{i}$ and $t_{i}$. $\Pi_{k}^{\prime}$ is well known to be NP-complete in general digraph even if $k=0$ (see Fortune, Hopcroft \& Wyllie (1980), Garey \& Johnson (1979)).

For a given $k$, we show the NP-completeness of $\Pi_{k}$, by exhibiting the following polynomial reduction from $\Pi_{k}^{\prime}$ to $\Pi_{k}$ :

For any instance of $\Pi_{k}^{\prime}$, considering a $k+1$-uplet $\left(\theta_{1}, \theta_{2}, \ldots, \theta_{k+1}\right)$ such that $\theta_{i} \in S_{k}$ or $\theta_{i} \in A_{k}$ and $i=1, \ldots, k+1$, its corresponding instance of $\Pi_{k}$ is obtained:

- by adding a vertex $\alpha_{i}$ and the $\operatorname{arcs}\left(t_{i}, \alpha_{i}\right)$ and $\left(\alpha_{i}, s_{i+1}\right)$, if $\theta_{i}$ is the vertex $\alpha_{i}$;

- by adding the $\operatorname{arcs}\left(\alpha_{i}, \beta_{i}\right),\left(t_{i}, \alpha_{i}\right)$ and $\left(\beta_{i}, s_{i+1}\right)$, if $\theta_{i}$ is the $\operatorname{arc}\left(\alpha_{i}, \beta_{i}\right)$;

- and by setting $s=s_{1}$ and $t=t_{k+2}$.

Lemma 2.1. The answer to the instance of $\Pi_{k}^{\prime}$ is YES iff the answer to the instance of $\Pi_{k}$ is also YES.

Proof. i) $\Rightarrow$ : Let $P_{s, t}$ be a path that visits the nodes $\alpha_{1}, \alpha_{2}, \ldots \alpha_{k+1}$ (in this order) in the graph of the instance of $\Pi_{k}$. This path can be decomposed into the sub-paths $P_{s_{i}, t_{i}}, P_{t_{i}, s_{i+1}}$ and $P_{s_{i+1}, t_{i+1}}, i=1, \ldots, k+1$ where $s=s_{1}, t=t_{k+2}$ and paths $P_{t_{i}, s_{i+1}}, i=1, \ldots, k+1$ are the sequences $t_{i}, \alpha_{i}, s_{i+1} . P_{s, t}$ cannot be elementary because $V\left(P_{s_{1}, t_{1}}\right) \cap \ldots \cap V\left(P_{s_{k+2}, t_{k+2}}\right) \neq \emptyset$, since the answer to the instance of $\Pi_{k}^{\prime}$ is YES.

ii $) \Leftarrow$ : Let $P_{s_{1}, t_{1}}, P_{s_{2}, t_{2}}, \ldots, P_{s_{k+2}, t_{k+2}}$ be paths in the graph of the instance of $\Pi_{k}^{\prime}$. Consider a path $P_{s, t}$ in the graph of the instance of $\Pi_{k} . P_{s, t}$ can be decomposed into the sub-paths $P_{s_{i}, t_{i}}$, $P_{t_{i}, s_{i+1}}$ and $P_{s_{i+1}, t_{i+1}}, i=1, \ldots, k+1$ where $s=s_{1}, t=t_{k+2}$ and paths $P_{t_{i}, s_{i+1}}, i=1, \ldots, k+$ 1 are the sequences $t_{i}, \alpha_{i}, s_{i+1}$. Since the answer to the instance of $\Pi_{k}$ is YES, $P_{s, t}$ is not elementary. This implies that $V\left(P_{s_{1}, t_{1}}\right) \cap \ldots \cap V\left(P_{s_{k+2}, t_{k+2}}\right) \neq \emptyset$. 
Theorem. The decision problem $\Pi_{k}$ is NP-complete.

Proof. As the decision problem $\Pi_{k}^{\prime}$ is known to be NP-complete in general digraph even if $k=0$ (see Fortune, Hopcroft \& Wyllie (1980), Garey \& Johnson (1979)), by Lemma 2.1, it's obvious that the problem $\Pi_{k}$ is also NP-complete.

After such polynomial transformation, for a given $k \in \mathbb{N}$, we conclude that the problem of separation of valid inequalities of order $k$ is NP-hard as its associated decision problem $\Pi_{k}$ is NP-complete.

\section{SEPARATION PROBLEM IN THE CASES $k=0$ AND $k=1$}

\subsection{Separation problem in the case $k=0$}

In the case $k=0$, the decision problem associated to the separation problem of valid inequalities of order $0, \Pi_{0}$, is formulated as follow:

"Given a vertex $\alpha$ or an $\operatorname{arc}(\alpha, \beta)$ in $G$, is $\alpha$ a 0 -vertex or is $(\alpha, \beta)$ a 0 -arc w.r.t. vertices $s$ and $t ? "$

To answer the complexity issue, let us consider the problem $\Pi_{0}^{\prime}$ defined as:

"Given four distinct vertices $s_{1}, t_{1}, s_{2}, t_{2}$, are there no two elementary paths, $P_{s_{1}, t_{1}}$ and $P_{s_{2}, t_{2}}$ in G such that $V\left(P_{s_{1}, t_{1}}\right) \cap V\left(P_{s_{2}, t_{2}}\right) \neq \emptyset$ ?" $\Pi_{0}^{\prime}$ is well known to be NP-complete in general digraphs, see Fortune, Hopcroft \& Wyllie (1980), Garey \& Johnson (1979).

The NP-completeness of problem $\Pi_{0}$ is readily obtained by considering the following polynomial reduction from $\Pi_{0}^{\prime}$ to $\Pi_{0}$ :

For any instance of $\Pi_{0}^{\prime}$, the corresponding instance of $\Pi_{0}$ is obtained by adding a vertex $\alpha$, two $\operatorname{arcs}\left(t_{1}, \alpha\right)$ and $\left(\alpha, s_{2}\right)$, and by setting $s=s_{1}$ and $t=t_{2}$, or by adding the $\operatorname{arcs}(\alpha, \beta),\left(t_{1}, \alpha\right)$, $\left(\beta, s_{2}\right)$ and we set $s=s_{1}$ and $t=t_{2}$.

Lemma 3.1. The answer to the instance of $\Pi_{0}^{\prime}$ is YES iff the answer to the instance of $\Pi_{0}$ is YES.

Proof. $\quad i) \Rightarrow$ : Let $P_{s, t}$ be a path that visits node $\alpha$ in the graph of the instance of $\Pi_{0}$. This path can be decomposed into the sub-paths $P_{s_{1}, t_{1}}, P_{t_{1}, s_{2}}, P_{s_{2}, t_{2}}$, where $s=s_{1}, t=t_{2}$ and path $P_{t_{1}, s_{2}}$ is the sequence $t_{1}, \alpha, s_{2}$. $P_{s, t}$ cannot be elementary because $V\left(P_{s_{1}, t_{1}}\right) \cap V\left(P_{s_{2}, t_{2}}\right) \neq \emptyset$, since the answer to the instance of $\Pi_{0}^{\prime}$ is YES.

ii) $\Leftarrow$ : Let $P_{s_{1}, t_{1}}$ and $P_{s_{2}, t_{2}}$ be paths in the graph of the instance of $\Pi_{0}^{\prime}$. Consider a path $P_{s, t}$ in the graph of the instance of $\Pi_{0} . P_{s, t}$ can be decomposed into the sub-paths $P_{s_{1}, t_{1}}, P_{t_{1}, s_{2}}, P_{s_{2}, t_{2}}$, where $s=s_{1}, t=t_{2}$ and path $P_{t_{1}, s_{2}}$ is the sequence $t_{1}, \alpha, s_{2}$. Since the answer to the instance of $\Pi_{0}$ is YES, $P_{s, t}$ is not elementary. This implies that

$$
V\left(P_{s_{1}, t_{1}}\right) \cap V\left(P_{s_{2}, t_{2}}\right) \neq \emptyset .
$$


Example. Consider the next instance of $\Pi_{0}^{\prime}$, such that $s_{1}=6, s_{2}=5, t_{1}=4$, and $t_{2}=3$ :

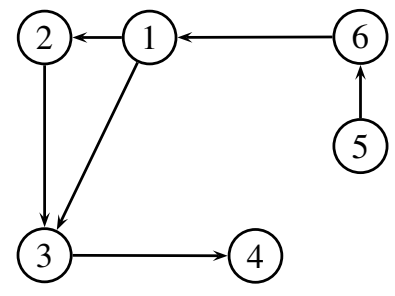

Figure 3 - An instance of $\Pi_{0}^{\prime}$.

The elementary paths between $s_{1}=6$ and $t_{1}=4$ represented by vertices $[6,1,3,4]$ and $[6,1,2,3,4]$ are not vertex-disjoints with $[5,6,1,2,3]$ and $[5,6,1,3]$ the elementary paths between $s_{2}=5$ and $t_{2}=3$, thus $\{6,1,3,4\} \cap\{5,6,1,2,3\} \neq \emptyset,\{6,1,2,3,4\} \cap\{5,6,1,2,3\} \neq \emptyset$, $\{6,1,3,4\} \cap\{5,6,1,3\} \neq \emptyset$ and $\{6,1,2,3,4\} \cap\{5,6,1,3\} \neq \emptyset$.

As explained above, to obtain the following instance of $\Pi_{0}$ from $\Pi_{0}^{\prime}$, we add the vertex $\alpha$ and the $\operatorname{arcs}(4, \alpha)$ and $(\alpha, 5)$ or by adding the $\operatorname{arcs}(\alpha, \beta),(4, \alpha)$ and $(\beta, 5)$
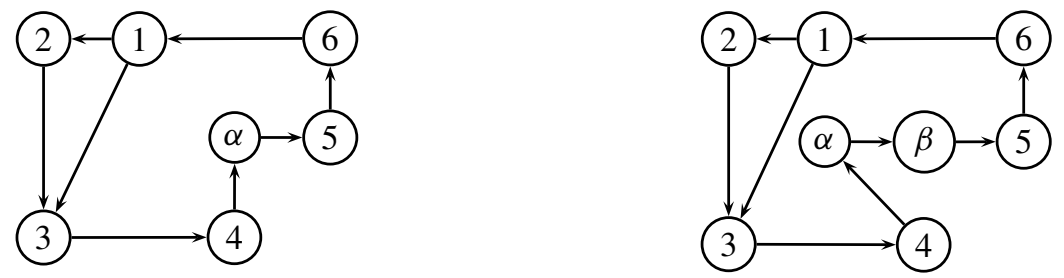

Figure 4 - The corresponding instance $\Pi_{0}$ of $\Pi_{0}^{\prime}$.

The fact that the answer of the problem $\Pi_{0}^{\prime}$ is YES, i.e, elementary paths represented by vertices $[6,1,3,4],[6,1,2,3,4]$ and $[5,6,1,3],[5,6,1,2,3]$ are not vertex-disjoints, it follows that the answer of the problem $\Pi_{0}$ is also YES. Then, $\alpha$ is a $0-$ vertex. One can observe that $\alpha$ does not belong to any elementary path between vertices $s_{1}=6$ and $t_{2}=3$ (see Fig. 4).

\subsection{Separation problem in the case $k=1$}

In the case $k=1$, the associate decision problem $\Pi_{1}$ is as follow: "Given two vertices $\alpha, \beta$ in $G$, are $\alpha$ and $\beta$ being 1-vertices w.r.t $s$ and $t$ ?". Consider the problem $\Pi_{1}^{\prime}$ :

"Given six distinct vertices $s_{1}, t_{1}, s_{2}, t_{2}, s_{3}, t_{3}$, are there no three elementary paths, $P_{s_{1}, t_{1}}, P_{s_{2}, t_{2}}$ and $P_{s_{3}, t_{3}}$ in G such that $V\left(P_{s_{i}, t_{i}}\right) \cap V\left(P_{s_{j}, t_{j}}\right)=\emptyset, 1 \leq i<j \leq 3$ ?"

As $\Pi_{0}^{\prime}$ is a special case of $\Pi_{1}^{\prime}$. $\Pi_{1}^{\prime}$ is NP-complete in general digraph, (see Fortune, Hopcroft \& Wyllie (1980), Garey \& Johnson (1979)). We show the NP-completeness of $\Pi_{1}$, by exhibiting the following polynomial reduction from $\Pi_{1}^{\prime}$ to $\Pi_{1}$ : For any instance of $\Pi_{1}^{\prime}$, the corresponding instance of $\Pi_{1}$ is obtained by adding the vertices $\alpha, \beta$ and the four arcs $\left(t_{1}, \alpha\right),\left(\alpha, s_{2}\right),\left(t_{2}, \beta\right)$ and $\left(\beta, s_{3}\right)$, and by setting $s=s_{1}$ and $t=t_{3}$. 
W.r.t arcs $\left(\alpha_{1}, \alpha_{2}\right)$ and $\left(\beta_{1}, \beta_{2}\right)$ to create the corresponding instance of $\Pi_{1}$ from any instance of $\Pi_{1}^{\prime}$, we add the $\operatorname{arcs}\left(\alpha_{1}, \alpha_{2}\right),\left(\beta_{1}, \beta_{2}\right),\left(t_{1}, \alpha_{1}\right),\left(\alpha_{2}, s_{2}\right),\left(t_{2}, \beta_{1}\right)$ and $\left(\beta_{2}, s_{3}\right)$.

Lemma 3.2. The answer to the instance of $\Pi_{1}^{\prime}$ is YES iff the answer to the instance of $\Pi_{1}$ is YES.

Proof. $i) \Rightarrow$ : Let $P_{s, t}$ be a path that visits the nodes $\alpha$ and $\beta$ (in this order) in the graph of the instance of $\Pi_{1}$. This path can be decomposed into the sub-paths $P_{s_{1}, t_{1}}, P_{t_{1}, s_{2}}, P_{s_{2}, t_{2}}, P_{t_{2}, s_{3}}$, $P_{s_{3}, t_{3}}$, where $s=s_{1}, t=t_{3}$ and path $P_{t_{1}, s_{2}}$ is the sequence $t_{1}, \alpha, s_{2}$ and path $P_{t_{2}, s_{3}}$ is the sequence $t_{2}, \beta, s_{3} . \quad P_{s, t}$ cannot be elementary because $V\left(P_{s_{1}, t_{1}}\right) \cap V\left(P_{s_{2}, t_{2}}\right) \cap V\left(P_{s_{3}, t_{3}}\right) \neq \emptyset$, since the answer to the instance of $\Pi_{1}^{\prime}$ is YES.

ii) $\Leftarrow$ : Let $P_{s_{1}, t_{1}}, P_{s_{2}, t_{2}}$ and $P_{s_{3}, t_{3}}$ be paths in the graph of the instance of $\Pi_{1}^{\prime}$. Consider a path $P_{s, t}$ in the graph of the instance of $\Pi_{1}$. $P_{s, t}$ can be decomposed into the sub-paths $P_{s_{1}, t_{1}}, P_{t_{1}, s_{2}}, P_{s_{2}, t_{2}}$, $P_{t_{2}, s_{3}}$ and $P_{s_{3}, t_{3}}$, where $s=s_{1}, t=t_{3}$ and path $P_{t_{1}, s_{2}}$ is the sequence $t_{1}, \alpha, s_{2}$ and path $P_{t_{2}, s_{3}}$ is the sequence $t_{2}, \beta, s_{3}$. Since the answer to the instance of $\Pi_{1}$ is YES, $P_{s, t}$ is not elementary. This implies that $V\left(P_{s_{1}, t_{1}}\right) \cap V\left(P_{s_{2}, t_{2}}\right) \cap V\left(P_{s_{3}, t_{3}}\right) \neq \emptyset$.

Example. Consider the below instance of $\Pi_{1}^{\prime}$ :

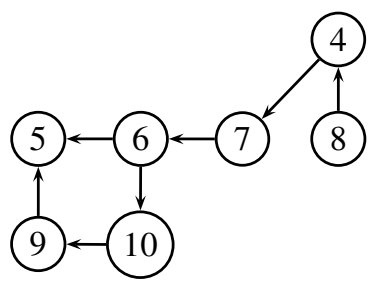

Figure 5 - An instance of $\Pi_{1}^{\prime}$.

Let $s_{1}=8, t_{1}=6, s_{2}=7, t_{2}=5, s_{3}=4$, and $t_{3}=10$, we observe that there is no three vertex-disjoint elementary paths between $\left(s_{1}, t_{1}\right),\left(s_{2}, t_{2}\right)$ and $\left(s_{3}, t_{3}\right)$ in the above instance of digraph. By transformation, we can obtain in polynomial time an instance of $\Pi_{1}$ as follow:
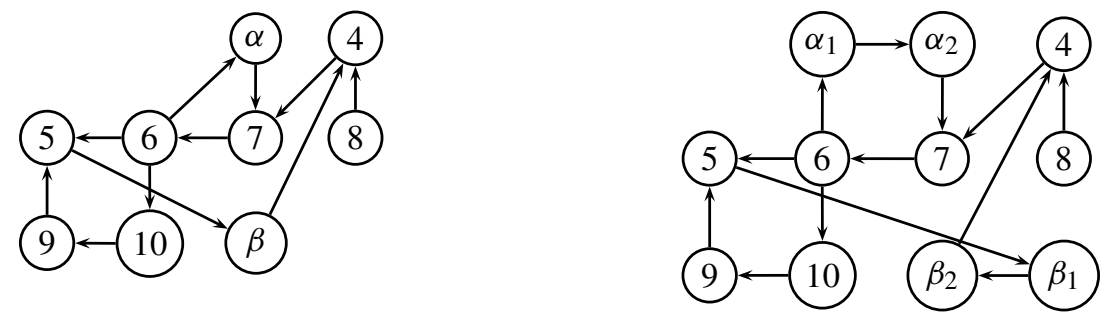

Figure 6 - The corresponding instance $\Pi_{1}$ of $\Pi_{1}^{\prime}$.

The fact that the answer of the problem $\Pi_{1}^{\prime}$ is YES, i.e, there is no vertex-disjoint elementary paths between vertices $(8,6),(7,5)$ and $(4,10)$ in the considered instance of $\Pi_{1}^{\prime}$, it follows that the answer of the problem $\Pi_{1}$ is also YES. Thus, it doesn't exist any elementary path between vertices $s_{1}=8$ and $t_{3}=10$ containing both vertices $\alpha$ and $\beta$ or $\operatorname{arcs}\left(\alpha_{1}, \alpha_{2}\right)$ and $\left(\beta_{1}, \beta_{2}\right)$. 


\section{CONCLUSION}

In this paper, we prove the NP-hardness of the separation problem of the so-called valid inequalities of order $k$. We establish a polynomial reduction from the problem of the existence of $k+2$ vertex-disjoint paths between $k+2$ pairs of vertices $\left(s_{1}, t_{1}\right),\left(s_{2}, t_{2}\right) \ldots\left(s_{k+2}, t_{k+2}\right)$ in a digraph to the decision problem associated to the separation of valid inequalities of order $k$. We recall that the problem of the existence of $k+2$ vertex-disjoint paths between $k+2$ pairs of vertices $\left(s_{1}, t_{1}\right),\left(s_{2}, t_{2}\right) \ldots\left(s_{k+2}, t_{k+2}\right)$ in a digraph is known to be NP-complete.

\section{ACKNOWLEDGMENTS}

We gratefully acknowledge the referees for their careful reading and insightful and constructive comments.

\section{REFERENCES}

[1] Fortune S, Hopcroft J \& Wyllie J. 1980. The directed subgraph homeomorphism problem. Theoretical Computer Science, 10: 111-121.

[2] GAREY M \& Johnson D. 1979. Computer and Intractibility: a guide to the theory of NPcompleteness. Freeman, San Francisco.

[3] IbRAhim MS. 2008. Etude de formulations et inégalités valides pour le problème du plus court chemin dans un graphe avec des circuits absorbants. PhD dissertation, LIP6, Université Pierre et Marie Curie, Paris, France.

[4] Ibrahim MS, Maculan N \& Minoux M. 2009. Strong flow-based formulation for the shortest path problem in digraphs with negative cycles. International Transaction in Operations Research (ITOR), 16: 361-369.

[5] Ibrahim MS, MaCUlan N \& Minoux M. 2014. Valid inequalities and lifting procedures for the shortest path problem in digraphs with negative cycles. Accepted to be published in Optimization Letters.

[6] Maculan N, Plateau G \& Lisser A. 2003. Integer linear models with a polynomial number of variables and constraints for some classical combinatorial optimization problems. Pesquisa Operacional, 23: 161-168.

[7] Ohtsuki T. 1981. The two disjoint path problem and wire routing design. Proceeding Symposium On Graph Theory and Applications, Lecture Notes in Computer Science, 108: 207-216, Berlin.

[8] Perl Y \& ShILOACH Y. 1978. Finding two disjoint paths between two pairs of vertices in a graph. J. ACM, 25: 1-9.

[9] Robertson N \& Seymour PD. 1995. Graphs minors XIII, The disjoint paths problem. J. Combinatorial Theory, Ser. B, 63: 65-110.

[10] SCHRIJVER A. 1994. Finding $k$-disjoint paths in directed planar graph. SIAM, J. Comput., 23: 780788.

[11] Seymour PD. 1980. Disjoint paths in graphs. Discrete Mathematics, 29: 293-309.

[12] SHILOACH Y. 1980. A polynomial solution to the undirected two paths problems. J. ACM, 27: 445456.

[13] Thomassen C. 1985. The two linkage problem for acyclic digraphs. Discrete Mathematics, 55: 73-87. 